\title{
Protocol for motor and language mapping by navigated TMS in patients and healthy volunteers; workshop report
}

\section{Krieg, Sandro M.}

2017-07

Krieg , S M , Lioumis , P , Mäkelä , J P , Wilenius , J , Karhu , J , Hannula , H, Savolainen , $P$, Weiss , C L , Seidel , K, Laakso , A , Islam , M , Vaalto , S , Lehtinen , H , Vitikainen , A-M , Tarapore , P E \& Picht , T 2017 , ' Protocol for motor and language mapping by navigated TMS in patients and healthy volunteers; workshop report ' , Acta Neurochirurgica , vol. 159 , no. 7 , pp. 1187-1195 . https://doi.org/10.1007/s00701-017-3187-z

http://hdl.handle.net/10138/237090

https://doi.org/10.1007/s00701-017-3187-z

publishedVersion

Downloaded from Helda, University of Helsinki institutional repository.

This is an electronic reprint of the original article.

This reprint may differ from the original in pagination and typographic detail.

Please cite the original version. 


\title{
Protocol for motor and language mapping by navigated TMS in patients and healthy volunteers; workshop report
}

\author{
Sandro M. Krieg ${ }^{1}$ - Pantelis Lioumis ${ }^{2}$ - Jyrki P. Mäkelä ${ }^{2}$. Juha Wilenius ${ }^{3}$. Jari Karhu ${ }^{4}$. \\ Henri Hannula ${ }^{4,5}$ • Petri Savolainen ${ }^{4}$. Carolin Weiss Lucas ${ }^{6} \cdot$ Kathleen Seidel $^{7}$. \\ Aki Laakso $^{8}$ - Mominul Islam ${ }^{9} \cdot$ Selja Vaalto $^{3}$ • Henri Lehtinen ${ }^{10}$. \\ Anne-Mari Vitikainen $^{2} \cdot$ Phiroz E. Tarapore ${ }^{11}$. Thomas Picht ${ }^{12}$
}

Received: 7 February 2017 / Accepted: 6 April 2017 / Published online: 29 April 2017

(C) Springer-Verlag Wien 2017

\begin{abstract}
Introduction Navigated transcranial magnetic stimulation (nTMS) is increasingly used for preoperative mapping of motor function, and clinical evidence for its benefit for brain tumor patients is accumulating. In respect to language mapping with repetitive nTMS, literature
\end{abstract}

\begin{tabular}{l}
\hline All authors contributed equally to this work \\
\hline The navigated TMS workshop group; Helsinki Meeting 2016 \\
\hline Electronic supplementary material The online version of this article \\
(doi:10.1007/s00701-017-3187-z) contains supplementary material, \\
which is available to authorized users.
\end{tabular}

Sandro M. Krieg

Sandro.Krieg@tum.de

Pantelis Lioumis

plioumis@gmail.com

Jyrki P. Mäkelä

Jyrki.Makela@hus.fi

Juha Wilenius

Juha.Wilenius@hus.fi

Jari Karhu

Jari.Karhu@nexstim.com

Henri Hannula

Henri.Hannula@nexstim.com

Petri Savolainen

Petri.Savolainen@nexstim.com

Carolin Weiss Lucas

Carolin.Weiss@uk-koeln.de

Kathleen Seidel

Kathleen.Seidel@insel.ch

Aki Laakso

Aki.Laakso@hus.fi reports have yielded variable results, and it is currently not routinely performed for presurgical language localization. The aim of this project is to define a common protocol for nTMS motor and language mapping to standardize its neurosurgical application and increase its clinical value.

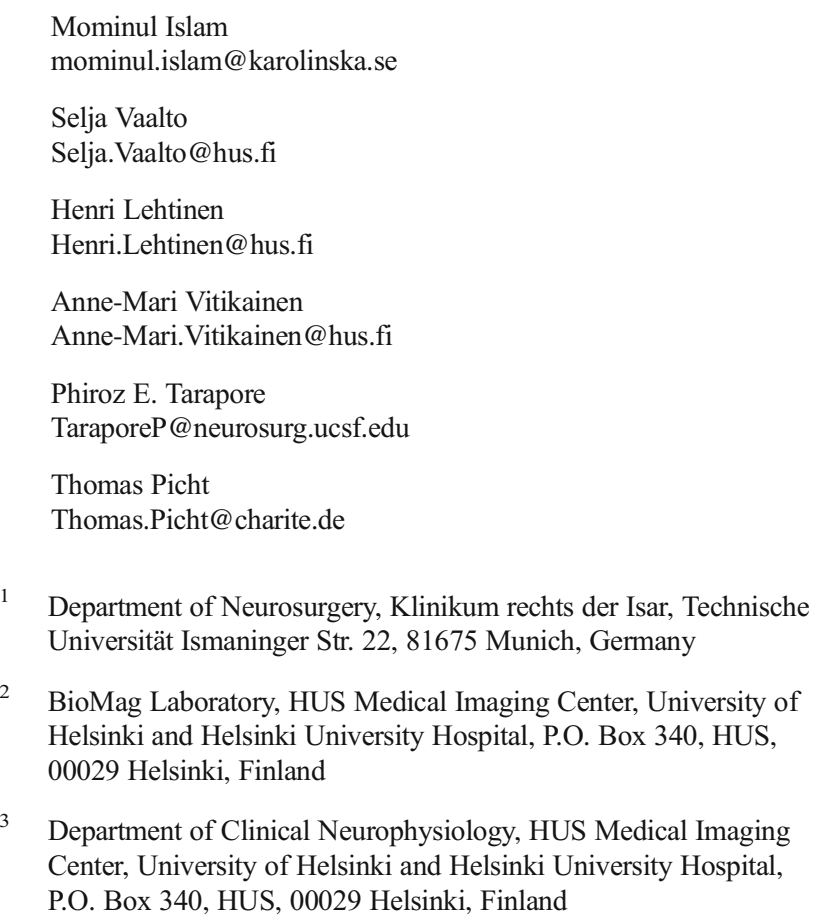
Helsinki and Helsinki University Hospital, P.O. Box 340, HUS, 00029 Helsinki, Finland

3 Department of Clinical Neurophysiology, HUS Medical Imaging Center, University of Helsinki and Helsinki University Hospital, P.O. Box 340, HUS, 00029 Helsinki, Finland 
Methods The nTMS workshop group, consisting of highly experienced nTMS users with experience of more than 1500 preoperative nTMS examinations, met in Helsinki in January 2016 for thorough discussions of current evidence and personal experiences with the goal to recommend a standardized protocol for neurosurgical applications.

Results nTMS motor mapping is a reliable and clinically validated tool to identify functional areas belonging to both normal and lesioned primary motor cortex. In contrast, this is less clear for language-eloquent cortical areas identified by nTMS. The user group agreed on a core protocol, which enables comparison of results between centers and has an excellent safety profile. Recommendations for nTMS motor and language mapping protocols and their optimal clinical integration are presented here.

Conclusion At present, the expert panel recommends nTMS motor mapping in routine neurosurgical practice, as it has a sufficient level of evidence supporting its reliability. The panel recommends that nTMS language mapping be used in the framework of clinical studies to continue refinement of its protocol and increase reliability.

Keywords Brain tumor $\cdot$ Epilepsy surgery $\cdot$ Motor . Language $\cdot$ Preoperative mapping $\cdot$ Transcranial magnetic stimulation

\section{Introduction}

Navigated transcranial magnetic stimulation (nTMS) has gained increasing acceptance for preoperative mapping of motor and language function in neurosurgical centers across the world [4, 11, 17, 26, 41, 47].

Clinical evidence of its benefit for the patients is growing $[10,19,26,38]$. Various protocols for motor and language mapping have been published to enhance the specificity and sensitivity of nTMS, [12, 13, 21, 22, 27, 30, 46]. Protocols for language mapping are particularly variable between centers $[14,21,22,42,45]$. This variability diminishes comparability of results and hampers its widespread adoption in clinical practice.

This workshop report aims to recommend a common protocol for nTMS mapping that addresses all aspects of its application, including the parameters used, data analyses, and clinical integration. It is a part of a larger project to establish a worldwide cooperation of researchers using nTMS for presurgical delineation of motor and language function and to fully integrate nTMS in clinical standards of care.

This report is not meant to replace existing guidelines on non-invasive stimulation of the brain [33]. It represents an adjunct for previous guidelines focusing on the presurgical use of nTMS in functional cortical mapping, particularly to benefit neurosurgeons and affiliated researchers.

Due to the extensive data already available for the use of nTMS in neurosurgery, this print article represents a shorter version of the original article, which is available as supplemental material to this report.

\section{The workshop meeting}

The nTMS workshop group, consisting of 11 experts with neurosurgical and scientific experience in nTMS motor and language mapping of up to 14 years, met in Helsinki, Finland, on 21-22 January 2016 and discussed current evidence and individual experiences to forge a recommended protocol for preoperative nTMS motor and language mappings.

To provide the best available evidence for the protocol, data from published clinical nTMS studies were used as a basis of these recommendations. Therefore, we reviewed the current literature via MEDLINE search and identified and analyzed all articles relevant for the use of nTMS in the neurosurgical field. The relevant articles for each aspect of nTMS mapping are cited in the particular paragraph.

For questions not dealt with in published reports, expert opinions were distilled to a common final statement. These recommendations, which cannot be proved to date by any available literature and are therefore based on our group consensus, do not possess any reference in their paragraph. 


\section{Methodological background on nTMS}

\section{Motor mapping}

Transcranial magnetic stimulation (TMS) is an electrophysiological technique for the investigation of the human motor cortex and motor tracts [1]. Development of TMS coils producing more focal cortical stimulation increased the spatial resolution of TMS. TMS delivers a short (rise time $100 \mu \mathrm{s}$ ), strong (1-2.7 T) magnetic field (pulse), which penetrates the skull and induces an electric field within the cortex. When appropriate stimulus intensity is used, this electric field activates cortical motoneurons of the corticospinal tract (CST). The descending corticospinal volley then excites $\alpha$ motoneurons of the anterior horn of the spinal cord generating a motor-evoked potential (MEP) in the muscle. The amplitude of the obtained MEP depends on stimulation intensity, coil shape and the quantity of excited motoneurons [7]. The efficacy of TMS is evaluated by continuous electromyography (EMG) monitoring to detect an MEP induced by stimulation of each cortical site comparable to intraoperative direct cortical stimulation (DCS) $[16,34,35]$.

\section{Language mapping}

In contrast to motor mapping, which uses single TMS pulses to elicit a MEP, language mapping protocols utilize repetitive TMS pulses (rTMS) [25]. As in DCS mapping during awake surgery, patients perform language tasks and rTMS is used to impair task performance by disrupting language-involved cortical regions $[6,8,44]$. The exact mechanisms by which rTMS interferes with language processing are not fully understood, but they likely involve a focal depolarization and temporary inhibition of neuronal networks involved in language processing [8].

\section{Neuronavigation}

When a TMS pulse is delivered, the affected cortical structure as well as the field strength within the structure depends on the individual brain anatomy (Fig. 1). By integrating a frameless stereotactic navigation system with the TMS coil and pulse generator, precise, real-time navigation and quantification of the magnetic field become possible. Current nTMS systems use a focal figure-of-eight TMS coil referenced to coordinates of the patient's head via an infrared tracking system. The induced electric field is visualized on the surface of the $3 \mathrm{D}$ head model that is reconstructed from the patient-specific MRI data. This technique, known as "navigated TMS" (nTMS), thereby enables exact delivery of a specific electric field to a given cortical structure $[16,34,35]$ and is a requirement for any pre-surgical mapping application.

\section{NTMS motor mapping}

\section{General information}

For more general information concerning clinical evidence, limitations of the method, troubleshooting, and patient selection, please see the supplemental material.

\section{Recommended protocol}

\section{Preparation}

An anatomical MRI with 1-mm slices is needed to create an adequate $3 \mathrm{D}$ brain reconstruction and enable accurate navigated coil positioning during nTMS mapping. In addition, a DWI data set for white matter tracking should be acquired to enable visualization of the cortico-subcortical connectivity.

\section{Selection of the monitored muscles}

Please see the supplemental material for a detailed list of used muscles for electrode placement. Most importantly, the muscles are chosen according to the tumor location and surgical approach planning.

\section{Mapping intensity}

Intensities slightly above the resting motor threshold (RMT) limit the cortical volume of stimulation and produce the most precise functional maps. For practical reasons, the use of the RMT of one small hand muscle (e.g., first dorsal interosseus; FDI) is used for the mapping of all upper extremity muscle representations. For mapping of cortical representations of the lower extremity and facial muscles, the stimulation intensity usually needs to be adapted (see below).

\section{FDI hot spot}

The functional hand motor area can approximately be identified by the omega-shaped knob in the precentral gyrus [48]. The search for the hand (here: FDI) hotspot is started from the anatomically identified hand motor area within the precentral gyrus. The FDI and abductor digiti minimi muscle (ADM) are equally useful for calculation of the RMT [29] and as the first focus of mapping. The stimulator output is adjusted to induce an electrical field of $80-100 \mathrm{~V} / \mathrm{m}$ on the cortex $20-25 \mathrm{~mm}$ below the coil (approximately 35-40\% of the maximum stimulator output, MSO). A rough mapping is then performed with MEPs between 100 and $500 \mu \mathrm{V}$ and extended along the central sulcus (CS) medially and laterally using the same intensity until the MEPs disappear while the orientation is kept perpendicular to the CS. The site eliciting maximum FDI MEPs defines the FDI hot spot. 
Fig. 1 Electric field:

Visualization of the induced electric field including quantification of the induced electric field strength in $\mathrm{V} / \mathrm{m}$

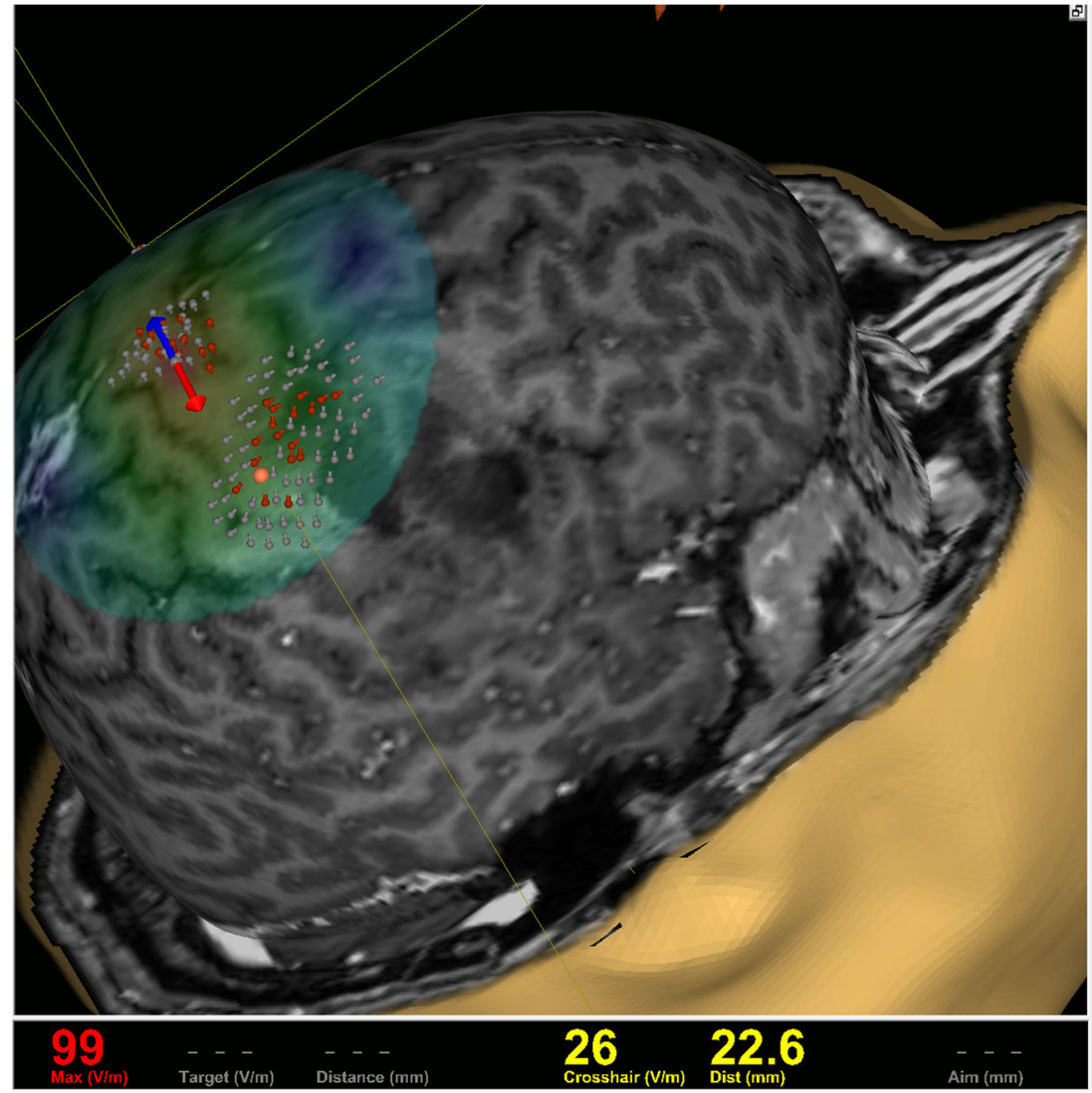

Resting motor threshold measurement

The RMT is defined as the lowest TMS intensity capable of eliciting a 50- $\mu \mathrm{V}$ MEP amplitude (peak to peak) in a relaxed small hand muscle (FDI or ADM) in five out of ten stimulations [32].

\section{Mapping of the hand motor area}

To optimize accuracy, the mapping should be carried out at the lowest possible intensity and done as described above for hot spot identification (stimulation intensity: 105\% RMT of FDI). An investigation of motor mapping approaches was performed by Raffin and colleagues; their paper describes in greater detail the theoretical background of the recommendations provided here and in the supplementary material [29].

\section{Mapping of lower extremity motor area}

The cortical representations of most leg and foot muscles are located deep within the interhemispheric fissure. Consequently, higher nTMS intensities are needed to elicit MEPs from the foot than from the hand muscles (Fig. 2) $[20,28]$.
Mapping of the facial motor area

Mapping of the facial motor area is outlined in the supplemental material.

\section{Utilization of mapping data for surgical planning}

All nTMS-positive cortical sites should be exported to the neuronavigation and can then be used as starting points for (somatotopic) white matter tracking via nTMS-based fiber tracking (FT) $[4,11,17,47]$. In addition to the TMS-derived topographical information, patient counseling and risk-benefit balancing can be supported by the RMT ratio of the hemisphere ipsilateral to the tumor divided by that of the contralateral hemisphere. The ratios above $110 \%$ or below $90 \%$ indicate an increased risk for postoperative motor deterioration [31]. During surgery the TMS maps can then be used to identify the motor cortex and guide cortical and subcortical electrical mapping [18].

\section{NTMS language mapping}

For more general information concerning clinical evidence, limitations of the method, troubleshooting, and patient selection, please see the supplemental material. 
Fig. 2 Motor mapping of the leg area: This graph shows a guide to obtaining optimal results for motor mapping of cortical leg areas
MAPPING THE LEG AREA (Tibialis anterior muscle (TA))

Coil orientation perpendicular to central fissure towards hand area at the junction of central sulcus and central fissure. Continue stimulation by following the central fissure 2 $\mathrm{cm}$ anteriorly and $2 \mathrm{~cm}$ posteriorly. Stimulate also perpendicular to the uppermost part of central sulcus, $3 \mathrm{~cm}$ from the central fissure.

\section{FINDING THE LEG RESPONSE}

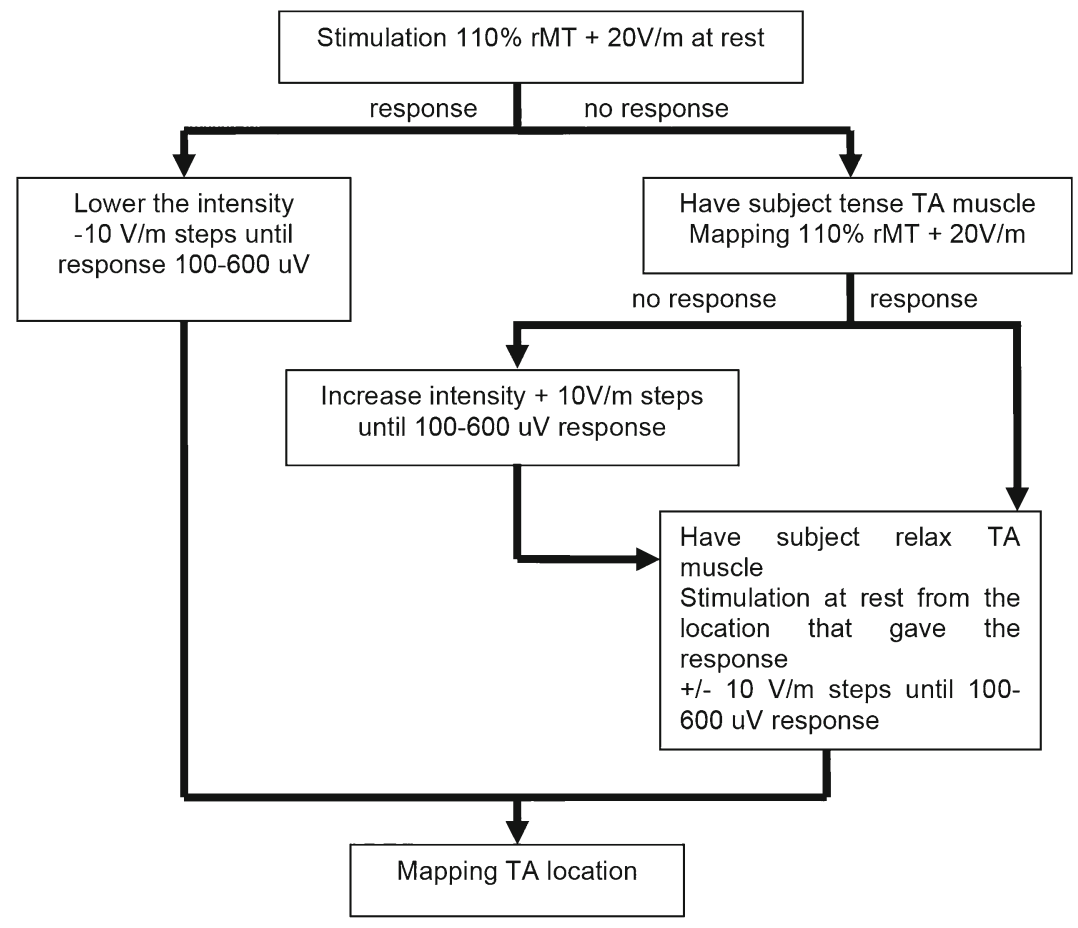

\section{Recommended protocol}

\section{Preparation}

Preparation for rTMS language mapping is identical to that required for motor mapping with special emphasis of the necessity of focused attention throughout the procedure.

\section{Language task}

The content and complexity of the task during language mapping affect the incidence, location, and type of observed errors [9]. As for intraoperative language mapping during awake surgery, the most frequently used task is confrontational object-naming with or without a written lead-in phrase. This task is well tolerated, fits within the time and space requirements, and also interrogates multiple aspects of language and speech. No other task has been proven superior for general mapping of languageeloquent brain areas [13-15].

\section{Baseline and rTMS object-naming}

The image set should contain only objects without synonyms to prevent erroneous interpretation of subject performance. Moreover, it needs to be presented in full to the patient at least two times before actual rTMS mapping ("baseline naming") while all misnamed images are be discarded. During the actual naming task, the remaining images should be presented timelocked to trains of TMS pulses, and the stimulation coil should be moved randomly between the presentations of the images in roughly $10-\mathrm{mm}$ steps over the perisylvian cortex. The induced current should be oriented perpendicular to the sulcus close to the stimulated point to induce a maximum effect. Table 1 shows an overview on the currently used rTMS setting of language mappings in the involved centers of the authors.

The following default parameters are recommended:

1. The baseline is repeated two to three times

2. Interpicture interval (IPI): $2500 \mathrm{~ms}$

3. Picture presentation time (PPT): $700 \mathrm{~ms}$

4. Stimulation intensity: ipsilateral RMT 
Table 1 Language mapping parameters: This table shows the variability of stimulation parameters as recommended by the different groups

\begin{tabular}{llllll}
\hline Group & Initial sentence & $\begin{array}{l}\text { Stimulus onset } \\
\text { delay (ms) }\end{array}$ & Frequency (Hz) & Pulses & $\begin{array}{l}\text { Picture presentation } \\
\text { time (ms) }\end{array}$ \\
\hline 1 & Yes & 0 & n.r. & n.r. & 500 \\
2 & No & 0 & 5 or 7 & 5 or 7 & 700 \\
3 & No & 0 & 7 & 7 & 700 \\
4 & No & 150 & 5 or 7 & 5 & 700 \\
5 & No & 0 & 5 or 7 & 5 or 7 & 700 \\
6 & Yes & 0 & 5 or 7 & 5 or 7 & 700 \\
7 & No & 150 & 5 or 7 or $\ldots .$. & 5 or 7 & 700 \\
8 & Yes & 300 & 7 & 7 & 700 \\
9 & No; but in pts. yes & 0 & 7 & 7 & 700 \\
\hline
\end{tabular}

n.r. $=$ no recommendation
5. Duration and frequency of the stimulation: $5 \mathrm{~Hz} / 5$ pulses

6. Picture to rTMS trigger interval (PTI): 0 ms delay [21,39]

7. The same cortical patch is repeated non-consecutively at least three times per mapping

During the examination, it is of utmost importance that the patient is comfortable and maintains a consistent level of attention. Video material demonstrating the language mapping procedure is available in the supplementary material of publications by the authors $[14,22]$.

\section{Result analysis and interpretation}

The enormous complexity of the cortico-subcortical network involved in the perception, processing, and production of language makes it a difficult target for single-trial mapping. The stimulation frequencies and the delivered electrical charge used in rTMS speech mapping differ significantly from DCS, and a single rTMS train does not give conclusive information about the language involvement of any particular cortical site. Successful rTMS language mapping is based on the application of several stimulation trains within the same cortical area and meticulous observation and documentation of the rTMS-induced behavioral changes. This analysis is only possible by video-based off-line review [22]. Clusters of sites inducing language disturbances within the same cortical area suggest that this area is relevant for speech processing. Interpretation of the rTMS language mapping results requires experience and careful consideration of each individual case.

\section{Stepwise description of the procedure}

This paragraph is only provided in the supplementary material.

\section{Result analysis}

Detection of subtle disturbances is achieved by comparing the baseline response to the response during rTMS stimulation $[5$, 27].

\section{Result interpretation}

It is crucial to realize that rTMS does not provide the same powerful blocking stimulus as DCS. Thus, ideally, a language specialist/neuropsychologist should be involved in the analysis of the language tasks.

\section{Utilization of mapping data for surgical planning}

The current experience and evidence suggest that cortical areas repeatedly targeted with no language disturbances induced by rTMS are most likely not carrying out essential language functions [21]. Clustering of rTMS-induced language disturbances within a distinct cortical area suggests relevant involvement in language processing, and DCS confirmation is recommended. Moreover, the combination of rTMS-positive cortical spots with tractography of subcortical language pathways via diffusion tensor imaging fiber tracking (DTI-FT) can aid in the interpretation of the results and add important information pertaining to the location of relevant cortical and subcortical structures. Protocols for rTMS-based DTI-FT have been described and compared to existing protocols $[24,40]$.

\section{Use of nTMS data in the operating room}

nTMS-positive sites for language and motor functions should be imported into the neuronavigation and hospital picture archiving system [23]. The DICOM standard supports this procedure. It is useful to export nTMS-positive stimulation points 


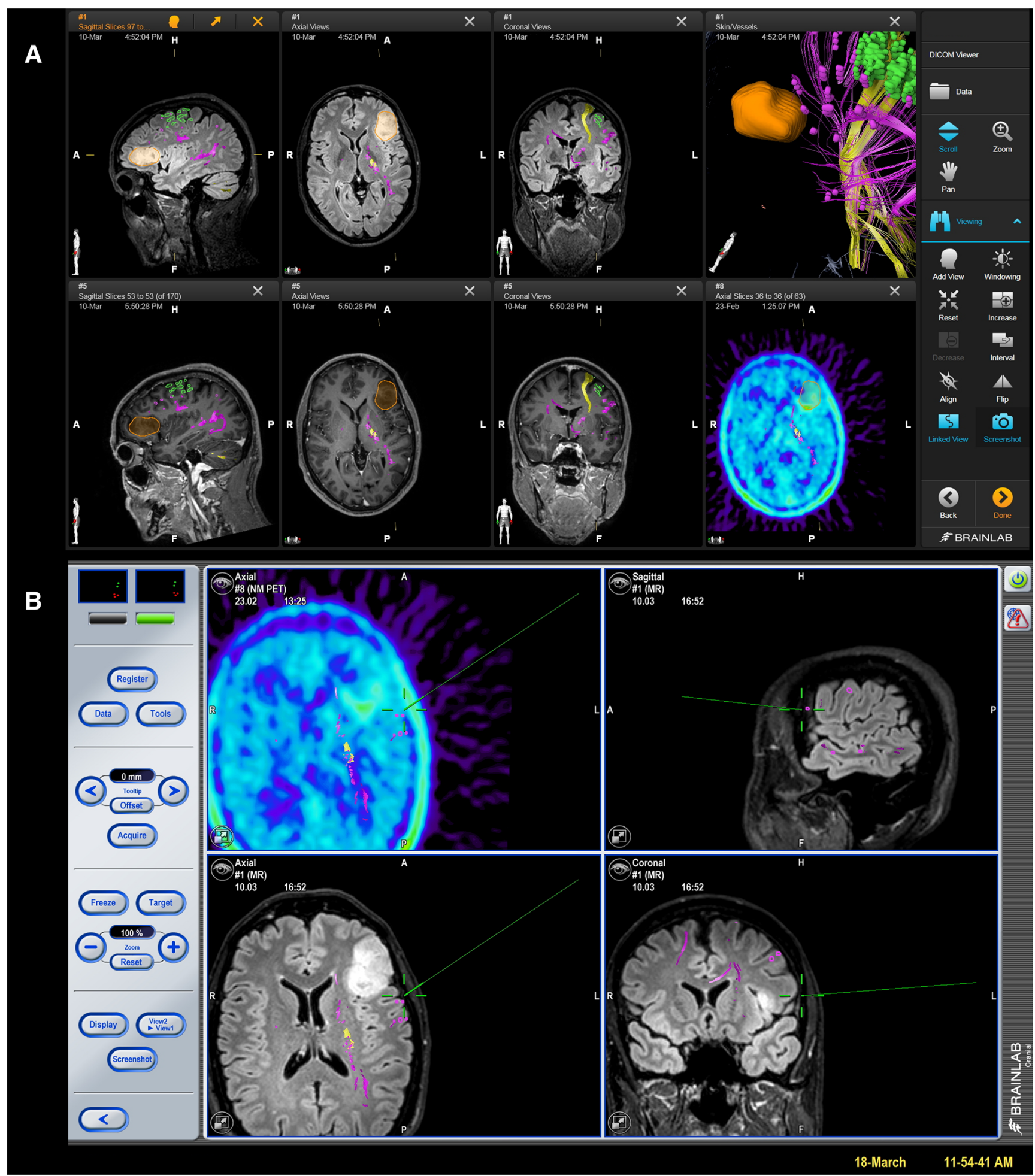

Fig. 3 Example of standardized color-coding: The screenshot shows a case of a left-sided oligodendroglioma WHO grade III located in the triangular part of the inferior frontal gyrus that was primarily judged by another neurosurgical department to be not resectable. This case gives an example of how nTMS can falsify presumed eloquence. Moreover, these

on various cortical and subcortical levels (i.e., peeling depths) to inform the surgeon. A standard color-coding of the screenshots show how to standardize different colors for the cortical motor areas (green), corticospinal tract (yellow), cortical language areas (purple), and subcortical language-related fiber tracts (purple). This allows an optimal preoperative preparation (A) and intraoperative clarification of the functional anatomy $(\mathrm{B})$

implemented rTMS data should be established to aid interpretation of the depicted functional anatomy and intraoperative 
use of the rTMS data (Fig. 3). The combination of rTMS results with rTMS-based FT to visualize language-related subcortical fibers and the CST is recommended. For language and motor eloquent lesions, however, final surgical decisions regarding the functional relevance of both cortical tissue and subcortical fiber tracts are made based on the intraoperative mapping and monitoring results $[2,3,36,37]$.

\section{Safety issues}

The protocols described here have been used for many years at the institutions of the authors. A safety analysis of these protocols revealed no major adverse events in 733 patients and 258 healthy subjects $[42,43]$.

\section{Conclusion and outlook}

nTMS motor mapping displays excellent accuracy in comparison with DCS, and the level of evidence is sufficient to recommend it for routine neurosurgical work-up. nTMS can be a valuable adjunct for established IOM workflows when planning and performing surgery in presumed motor eloquent areas.

The workshop group recommends nTMS language mapping in the frame of clinical studies but not to replace awake surgery for eloquent lesions. Moreover, development of experimental protocols should be a priority for clinicians interested in the management of lesions in eloquent brain regions. The group members involved in these recommendations will use the common protocols as they have been described and will compare the results in upcoming meetings.

\section{Compliance with ethical standards}

Funding Nexstim Plc. (Helsinki, Finland) provided financial support in the form of travel funding to the venue site at BioMag Laboratory, HUS Medical Imaging Center, University of Helsinki and Helsinki University Hospital, Helsinki, Finland. The sponsor had no role in the design or conduct of this report.

Conflicts of interest SK and TP are consultants for Brainlab AG (Munich, Germany) and for Nexstim Plc. (Helsinki, Finland). PL is consultant for Nexstim Plc. HH, PS, and JK are employed by Nexstim Plc. Yet, all authors report no conflict of interest concerning the materials or methods used in this study or the findings specified in this paper.

Ethical approval Since this is a report from an expert panel, no ethics approval was obtained. This article does not contain any studies with human participants performed by any of the authors.

\section{References}

1. Barker AT, Jalinous R, Freeston IL (1985) Non-invasive magnetic stimulation of human motor cortex. Lancet 1:1106-1107

2. Bello L, Gallucci M, Fava M, Carrabba G, Giussani C, Acerbi F, Baratta P, Songa V, Conte V, Branca V, Stocchetti N, Papagno C, Gaini SM (2007) Intraoperative subcortical language tract mapping guides surgical removal of gliomas involving speech areas. Neurosurgery 60:67-80

3. Bello L, Riva M, Fava E, Ferpozzi V, Castellano A, Raneri F, Pessina F, Bizzi A, Falini A, Cerri G (2014) Tailoring neurophysiological strategies with clinical context enhances resection and safety and expands indications in gliomas involving motor pathways. Neuro-Oncology 16:1110-1128

4. Conti A, Raffa G, Granata F, Rizzo V, Germano A, Tomasello F (2014) Navigated transcranial magnetic stimulation for "somatotopic" tractography of the corticospinal tract. Neurosurgery 10(Suppl 4):542-554 discussion 554

5. Corina DP, Loudermilk BC, Detwiler L, Martin RF, Brinkley JF, Ojemann G (2010) Analysis of naming errors during cortical stimulation mapping: implications for models of language representation. Brain Lang 115:101-112

6. Devlin JT, Watkins KE (2007) Stimulating language: insights from TMS. Brain 130:610-622

7. Di Lazzaro V, Oliviero A, Pilato F, Saturno E, Insola A, Mazzone P, Tonali PA, Rothwell JC (2002) Descending volleys evoked by transcranial magnetic stimulation of the brain in conscious humans: effects of coil shape. Clin Neurophysiol 113:114-119

8. Epstein CM, Lah JJ, Meador K, Weissman JD, Gaitan LE, Dihenia B (1996) Optimum stimulus parameters for lateralized suppression of speech with magnetic brain stimulation. Neurology 47:1590 1593

9. Fernandez Coello A, Moritz-Gasser S, Martino J, Martinoni M, Matsuda R, Duffau H (2013) Selection of intraoperative tasks for awake mapping based on relationships between tumor location and functional networks. J Neurosurg 119:1380-1394

10. Frey D, Schilt S, Strack V, Zdunczyk A, Rosler J, Niraula B, Vajkoczy P, Picht T (2014) Navigated transcranial magnetic stimulation improves the treatment outcome in patients with brain tumors in motor eloquent locations. Neuro-Oncology 16:1365-1372

11. Frey D, Strack V, Wiener E, Jussen D, Vajkoczy P, Picht T (2012) A new approach for corticospinal tract reconstruction based on navigated transcranial stimulation and standardized fractional anisotropy values. NeuroImage 62:1600-1609

12. Hauck T, Tanigawa N, Probst M, Wohlschlaeger A, Ille S, Sollmann N, Maurer S, Zimmer C, Ringel F, Meyer B, Krieg SM (2015) Stimulation frequency determines the distribution of language positive cortical regions during navigated transcranial magnetic brain stimulation. BMC Neurosci 16:5

13. Hauck T, Tanigawa N, Probst M, Wohlschlaeger A, Ille S, Sollmann N, Maurer S, Zimmer C, Ringel F, Meyer B, Krieg SM (2015) Task type affects location of language-positive cortical regions by repetitive navigated transcranial magnetic stimulation mapping. PLoS One 10:e125298

14. Hernandez-Pavon JC, Makela N, Lehtinen H, Lioumis P, Makela JP (2014) Effects of navigated TMS on object and action naming. Front Hum Neurosci 8:660

15. Hervey-Jumper SL, Li J, Lau D, Molinaro AM, Perry DW, Meng L, Berger MS (2015) Awake craniotomy to maximize glioma resection: methods and technical nuances over a 27 -year period. J Neurosurg 123:325-339

16. Ilmoniemi RJ, Ruohonen J, Karhu J (1999) Transcranial magnetic stimulation - a new tool for functional imaging of the brain. Crit RevBiomedEng 27:241-284 
17. Krieg SM, Buchmann NH, Gempt J, Shiban E, Meyer B, Ringel F (2012) Diffusion tensor imaging fiber tracking using navigated brain stimulation - a feasibility study. Acta Neurochir 154:555-563

18. Krieg SM, Picht T, Sollmann N, Bährend I, Ringel F, Nagarajan SS, Meyer B, Tarapore PE (2016) Resection of motor eloquent metastases aided by preoperative nTMS-based motor maps - comparison of two observational cohorts. Frontiers in Oncology 6:261

19. Krieg SM, Sabih J, Bulubasova L, Obermueller T, Negwer C, Janssen I, Shiban E, Meyer B, Ringel F (2014) Preoperative motor mapping by navigated transcranial magnetic brain stimulation improves outcome for motor eloquent lesions. Neuro-Oncology 16:1274-1282

20. Krieg SM, Shiban E, Buchmann N, Gempt J, Foerschler A, Meyer B, Ringel F (2012) Utility of presurgical navigated transcranial magnetic brain stimulation for the resection of tumors in eloquent motor areas. J Neurosurg 116:994-1001

21. Krieg SM, Tarapore PE, Picht T, Tanigawa N, Houde J, Sollmann N, Meyer B, Vajkoczy P, Berger MS, Ringel F, Nagarajan S (2014) Optimal timing of pulse onset for language mapping with navigated repetitive transcranial magnetic stimulation. NeuroImage 100:219-236

22. Lioumis P, Zhdanov A, Makela N, Lehtinen H, Wilenius J, Neuvonen T, Hannula H, Deletis V, Picht T, Makela JP (2012) A novel approach for documenting naming errors induced by navigated transcranial magnetic stimulation. J Neurosci Methods 204:349-354

23. Makela T, Vitikainen AM, Laakso A, Makela JP (2015) Integrating nTMS data into a radiology picture archiving system. J Digit Imaging 28:428-432

24. Negwer C, Sollmann N, Ille S, Hauck T, Maurer S, Kirschke JS, Ringel F, Meyer B, Krieg SM (2016) Language pathway tracking: comparing nTMS-based DTI fiber tracking with a cubic ROIsbased protocol. J Neurosurg :1-9

25. Pascual-Leone A, Gates JR, Dhuna A (1991) Induction of speech arrest and counting errors with rapid-rate transcranial magnetic stimulation. Neurology 41:697-702

26. Picht T, Frey D, Thieme S, Kliesch S, Vajkoczy P (2016) Presurgical navigated TMS motor cortex mapping improves outcome in glioblastoma surgery: a controlled observational study. J Neuro-Oncol 126:535-543

27. Picht T, Krieg SM, Sollmann N, Rosler J, Niraula B, Neuvonen T, Savolainen P, Lioumis P, Makela JP, Deletis V, Meyer B, Vajkoczy P, Ringel F (2013) A comparison of language mapping by preoperative navigated transcranial magnetic stimulation and direct cortical stimulation during awake surgery. Neurosurgery 72:808-819

28. Picht T, Mularski S, Kuehn B, Vajkoczy P, Kombos T, Suess O (2009) Navigated transcranial magnetic stimulation for preoperative functional diagnostics in brain tumor surgery. Neurosurgery 65:93-98

29. Raffin E, Pellegrino G, Di Lazzaro V, Thielscher A, Siebner HR (2015) Bringing transcranial mapping into shape: sulcus-aligned mapping captures motor somatotopy in human primary motor hand area. NeuroImage 120:164-175

30. Rogic M, Deletis V, Fernandez-Conejero I (2014) Inducing transient language disruptions by mapping of Broca's area with modified patterned repetitive transcranial magnetic stimulation protocol. J Neurosurg 120:1033-1041

31. Rosenstock T, Grittner U, Acker G, Schwarzer V, Kulchytska N, Vajkoczy P, Picht T (2016) Risk stratification in motor area-related glioma surgery based on navigated transcranial magnetic stimulation data. J Neurosurg : 1-11

32. Rossini PM, Barker AT, Berardelli A, Caramia MD, Caruso G, Cracco RQ, Dimitrijevic MR, Hallett M, Katayama Y, Lucking $\mathrm{CH}$ et al (1994) Non-invasive electrical and magnetic stimulation of the brain, spinal cord and roots: basic principles and procedures for routine clinical application. Report of an IFCN committee. Electroencephalogr Clin Neurophysiol 91:79-92

33. Rossini PM, Burke D, Chen R, Cohen LG, Daskalakis Z, Di Iorio R, Di Lazzaro V, Ferreri F, Fitzgerald PB, George MS, Hallett M, Lefaucheur JP, Langguth B, Matsumoto $\mathrm{H}$, Miniussi C, Nitsche
MA, Pascual-Leone A, Paulus W, Rossi S, Rothwell JC, Siebner HR, Ugawa Y, Walsh V, Ziemann U (2015) Non-invasive electrical and magnetic stimulation of the brain, spinal cord, roots and peripheral nerves: basic principles and procedures for routine clinical and research application. An updated report from an IFCN Committee. Clin Neurophysiol 126:1071-1107

34. Ruohonen J, Ilmoniemi RJ (1999) Modeling of the stimulating field generation in TMS. Electroencephalogr Clin Neurophysiol Suppl $51: 30-40$

35. Ruohonen J, Karhu J (2010) Navigated transcranial magnetic stimulation. NeurophysiolClin 40:7-17

36. Seidel K, Beck J, Stieglitz L, Schucht P, Raabe A (2013) The warning-sign hierarchy between quantitative subcortical motor mapping and continuous motor evoked potential monitoring during resection of supratentorial brain tumors. J Neurosurg 118:287-296

37. Soffietti R, Baumert BG, Bello L, von Deimling A, Duffau H, Frenay M, Grisold W, Grant R, Graus F, Hoang-Xuan K, Klein M, Melin B, Rees J, Siegal T, Smits A, Stupp R, Wick W (2010) Guidelines on management of low-grade gliomas: report of an EFNS-EANO task force. Eur J Neurol 17:1124-1133

38. Sollmann N, Ille S, Hauck T, Maurer S, Negwer C, Zimmer C, Ringel F, Meyer B, Krieg SM (2015) The impact of preoperative language mapping by repetitive navigated transcranial magnetic stimulation on the clinical course of brain tumor patients. BMC Cancer 15:261

39. Sollmann N, Ille S, Negwer C, Boeckh-Behrens T, Ringel F, Meyer B, Krieg SM (2016) Cortical time course of object naming investigated by repetitive navigated transcranial magnetic stimulation. Brain Imaging Behav. doi:10.1007/s11682-11016-19574-x

40. Sollmann N, Negwer C, Ille S, Maurer S, Hauck T, Kirschke JS, Ringel F, Meyer B, Krieg SM (2016) Feasibility of nTMS-based DTI fiber tracking of language pathways in neurosurgical patients using a fractional anisotropy threshold. J Neurosci Methods 267:45-54

41. Tarapore PE, Findlay AM, Honma SM, Mizuiri D, Houde JF, Berger MS, Nagarajan SS (2013) Language mapping with navigated repetitive TMS: proof of technique and validation. NeuroImage $82: 260-272$

42. Tarapore PE, Picht T, Bulubas L, Shin Y, Kulchytska N, Meyer B, Berger MS, Nagarajan SS, Krieg SM (2016) Safety and tolerability of navigated TMS for preoperative mapping in neurosurgical patients. Clin Neurophysiol 127:1895-1900

43. Tarapore PE, Picht T, Bulubas L, Shin Y, Kulchytska N, Meyer B, Nagarajan SS, Krieg SM (2016) Safety and tolerability of navigated TMS in healthy volunteers. Clin Neurophysiol 127:1916-1918

44. Vigliocco G, Vinson DP, Druks J, Barber H, Cappa SF (2011) Nouns and verbs in the brain: a review of behavioural, electrophysiological, neuropsychological and imaging studies. Neurosci Biobehav Rev 35:407-426

45. Vitikainen AM, Makela E, Lioumis P, Jousmaki V, Makela JP (2015) Accelerometer-based automatic voice onset detection in speech mapping with navigated repetitive transcranial magnetic stimulation. J Neurosci Methods 253:70-77

46. Weiss C, Nettekoven C, Rehme AK, Neuschmelting V, Eisenbeis A, Goldbrunner R, Grefkes C (2013) Mapping the hand, foot and face representations in the primary motor cortex - retest reliability of neuronavigated TMS versus functional MRI. NeuroImage 66:531-542

47. Weiss C, Tursunova I, Neuschmelting V, Lockau H, Nettekoven C, Oros-Peusquens AM, Stoffels G, Rehme AK, Faymonville AM, Shah NJ, Langen KJ, Goldbrunner R, Grefkes C (2015) Improved nTMS- and DTI-derived CST tractography through anatomical ROI seeding on anterior pontine level compared to internal capsule. Neuroimage Clin 7:424-437

48. Yousry TA, Schmid UD, Alkadhi H, Schmidt D, Peraud A, Buettner A, Winkler P (1997) Localization of the motor hand area to a knob on the precentral gyrus. A new landmark. Brain 120(Pt 1): $141-157$ 